\title{
Les idées religieuses de George Sand et l'émancipation féminine
}

\section{Marie-Reine Renard}

\section{(2) OpenEdition}

12 Journals

Édition électronique

URL : http://journals.openedition.org/assr/1902

DOI : 10.4000/assr.1902

ISSN : $1777-5825$

Éditeur

Éditions de l'EHESS

\section{Édition imprimée}

Date de publication : 1 octobre 2004

Pagination : 25-38

ISBN : 2-222-96754-6

ISSN : 0335-5985

\section{Référence électronique}

Marie-Reine Renard, «Les idées religieuses de George Sand et l'émancipation féminine », Archives de sciences sociales des religions [En ligne], 128 | octobre - décembre 2004, mis en ligne le 19 septembre 2007, consulté le 02 mai 2019. URL : http://journals.openedition.org/assr/1902 ; DOI : 10.4000/ assr.1902 


\section{LES IDÉES RELIGIEUSES DE GEORGE SAND ET L'ÉMANCIPATION FÉMININE}

\section{Introduction}

La célébration du bicentenaire de la naissance de George Sand, née Aurore Dupin de Francueil le $1^{\text {er }}$ juillet 1804, morte le 8 juin 1876 donne lieu à de nombreuses manifestations, colloques, publications d'études, réédition de plusieurs ouvrages, sur lesquels fait le point Le Magazine Littéraire de mai 2004 (1). Son œuvre, particulièrement abondante, puisqu'elle comporte environ quatre-vingts romans, vingt-six volumes de correspondance, des études diverses, ainsi que des écrits autobiographiques, sans compter ses collaborations à de nombreux journaux, constitue un précieux reflet des préoccupations de son époque. George Sand devint célèbre grâce à la publication de ses premiers romans, Indiana, Valentine, Lélia, dont le thème était la condition des femmes mariées, et qui illustraient les inconvénients de leur statut matrimonial. Les théories de George Sand prenaient appui sur certains courants religieux de l'époque romantique, dont sortira également le socialisme dit «utopique ». À l'approche de l'année 2005, consacrée à la laïcité, laquelle peut s'analyser comme un moyen de concilier croyances et liberté religieuses, il est particulièrement intéressant de rechercher dans quelle mesure des convictions religieuses peuvent s'allier à des théories émancipatrices telles que le féminisme. Une telle étude doit permettre également de mesurer les limites intellectuelles posées à l'émancipation des femmes au cours du XIX ${ }^{\mathrm{e}}$ siècle. Il conviendra donc d'examiner dans une première partie les rapports qui existent entre les religiosités romantiques et le féminisme, puis en second lieu la synthèse qu'en élabora George Sand dans son œuvre.

(1) « George Sand, une rebelle face à son siècle », Le Magazine Littéraire 2004. Ce périodique est consacré à la commémoration du bicentenaire de la naissance de George Sand, et fait le point des colloques, expositions et parutions récentes qui lui sont consacrées. 


\section{Les religiosités romantiques et l'apparition du féminisme}

\section{Les sources religieuses du romantisme}

Il est acquis de longue date que le romantisme a pour racines des idées mystiques, théosophiques, qui apparaissent à la fin du XVIII siècle, d'abord en Angleterre et en Allemagne, et plus discrètement en France, où le mouvement fut décrit par Auguste Viatte dans un ouvrage intitulé Les sources occultes du romantisme (2). Il importe de noter que d'emblée, ce courant littéraire s'accompagne d'une reconsidération du rôle de la femme qui devient un guide, comme en témoignent à titre d'exemple certains écrits de Novalis (3) ou de Hoffmann (4). À la même époque furent publiés les premiers écrits féministes, en France sous les plumes de Condorcet et d'Olympe de Gouges, chez Mary Wollstonecraft en Angleterre, de von Hippel en Prusse (5). En 1812, Percy B. Shelley fait paraître un poème philosophique intitulé La Reine Mab, lequel critique les valeurs du monde patriarcal et place la société à venir sous l'égide d'une entité féminine, Mab, la reine des fées (6). Quelques décennies plus tard, George Sand publiera une ballade portant le même titre, mais qui n'empruntera à Shelley que le titre et quelques images (7).

Lorsque après les bouleversements consécutifs à la Révolution et à l'Empire, il apparût que l'ordre ancien ne pouvait être restauré, en dépit des tentatives faites en ce sens, nombre d'écrivains, de penseurs, s'employèrent à imaginer quel serait le monde qui émergerait de ces ruines. C'est ce que Paul Bénichou a appelé Le Temps des prophètes (8). Pour Pierre-Simon Ballanche (1776-1847), les drames consécutifs à la Révolution vont permettre la naissance d'une nouvelle société. C'est ce qu'il appelle la « palingénésie ». Saint-Simon (1760-1825), Auguste Comte (17981857), Hoëné Wronski (1768-1853) veulent fonder la société future sur la science. Toutefois, ils deviennent progressivement et à des degrés divers les prophètes de nouvelles religions. Du saint-simonisme sortiront à la fois le socialisme et le féminisme, ainsi que le démontre la thèse de Marguerite Thibert intitulée Le féminisme dans le socialisme français de 1830 à 1850 (9). Si Saint-Simon lui-même n'est l'auteur d'aucun écrit féministe, il prévoit l'avènement d'une nouvelle société, industrieuse et pacifique, dirigée par des savants, où rien ne fera obstacle à un rôle prépondérant des femmes. D'ailleurs, les premiers disciples de Saint-Simon, notamment Bazard et Enfantin, à la recherche de nouvelles solutions aux problèmes de leur temps, se laisseront séduire par les courants mystiques de leur époque et rejoindront pas ces voies le mouvement de divinisation de la femme (10). Dans

(2) Viatte, 1979 (1927).

(3) Novalis, « Heinrich von Ofterdingen » in Philippe BOYER, Le romantisme allemand, Paris, MA Éditions, 1985, p. 127. Article dans lequel le personnage de Mathilde doit conduire le poète jusqu'à l'Âge d'Or afin de retrouver la fleur bleue.

(4) Hoffmann, 1964, pp. 241-365.

(5) Abensour, 1979, pp. 187-190.

(6) SHELlEy, 1907, pp. 12-77.

(7) SAND, «La Reine Mab », in Légendes rustiques, 1975, pp. 165-168.

(8) Benichou, 1977.

(9) THibert, 1926, p. 9.

(10) Charlety, 1965, (1931), pp. 109-112. 
cette optique, la société sera régénérée grâce à la réhabilitation de deux catégories d'êtres jusqu'alors défavorisés : les femmes et les prolétaires. Flora Tristan (18031844), qui fréquente les cercles saint-simoniens, développera cette idée dans ses principaux ouvrages (11).

Ces idées de «réhabilitation», de «divinisation» de la femme vont se répandre et se retrouver sous de nombreuses plumes pendant la première moitié du $\mathrm{XIX}^{\mathrm{e}}$ siècle. Charles Fourier avait émis l'idée selon laquelle l'individu social est un couple formé d'un homme et d'une femme. À leur tour, les saint-simoniens vont affirmer qu'à côté du Père qui, en la personne d'Enfantin préside à leur mouvement, doit un jour se joindre une « Mère », et placent à cet effet un fauteuil vide à côté de celui du Père. Un personnage original, le sculpteur Ganneau, prêche dans les milieux artistiques une nouvelle religion dont il est le grand prêtre sous le nom de Mapah, formé de la première syllabe de «maman » et de «papa », pour marquer le caractère à la fois féminin et masculin de la nouvelle divinité. De même, il appelle sa doctrine «l'évadisme», formé de la contraction d'Ève et Adam. Le romantisme s'accompagne donc d'un retour des cultes féminins, en dehors bien entendu des religions établies. Mais d'autres auteurs vont s'attacher à donner une caution scientifique à ces théories encore diffuses.

\section{Les religions « amies du droit de la mère »}

Johann-Jakob Bachofen (1815-1887), d'abord professeur de droit à l'Université de Bâle, s'intéresse aux antiquités gréco-romaines, ce qui l'amène à découvrir une nouvelle signification aux mythologies païennes. Il expose ses thèses dans un gros ouvrage paru en 1861 intitulé Das Mutterrecht, «Le droit de la mère », dans lequel il démontre que la place de la femme dans la société n'a pas toujours été subordonnée à celle de l'homme, car les mythes des anciennes littératures mettent en scène des femmes qui luttent pour conquérir ou conserver le pouvoir (12), où encore, comme tend à le démontrer la légende de Tanaquil, confèrent la royauté à des hommes (13). De ces différentes observations, Bachofen tire la conclusion qu'il a existé des sociétés gynécocratiques, au sein desquelles règnent des valeurs différentes de celles du monde patriarcal. L'accent y est mis sur la vie matérielle et naturelle, de même que le langage des symboles fait appel à l'idée d'élément aquatique, à l'obscurité, etc... Le thème de la gynécocratie et de ses valeurs opposées à celles du monde patriarcal sera abondamment utilisé par les mouvements féministes au tournant des $\mathrm{XIX}^{\mathrm{e}}$ et $\mathrm{XX}^{\mathrm{e}}$ siècles (14). Bachofen met en évidence qu'il existe des religions « amies du droit de la mère ». Ces idées seront reprises par un auteur plus récent, Robert Graves.

Ce dernier publia en 1948 La Déesse blanche, qui pose la question des cultes féminins de l'Antiquité et de leurs survivances (15). Les conclusions de ces auteurs ont bien entendu appelé réserves et critiques, ne serait-ce que parce que l'étude des mythes et leur interprétation s'avère nécessairement délicate. S'il est vrai qu'à leur

(11) LEO, 1994, p. 48.

(12) BACHOFEN, 1993.

(13) BACHOFEN, 1980 (1938), pp. 109-110.

(14) BACHOFEN «Le droit de la mère dans l'Antiquité », (préface) in BACHOFEN, 1903.

(15) Graves, 1979. 
origine se trouvent le plus souvent quelques faits réels, ceux-ci ont pu être considérablement déformés et peuvent donner lieu à des interprétations divergentes. Léon Abensour, dans son Histoire Générale du féminisme en donne un bon exemple à propos de la lutte des héros grecs contre les Amazones, divinités féminines, lunaires: les adversaires de celles-ci sont des divinités solaires, masculines. Ce serait donc une lutte d'influence entre les dieux, et non pas la représentation, sur un mode allégorique de la lutte et du triomphe du patriarcat sur le matriarcat (16). Mais les intuitions de Bachofen frayaient la voie à certaines notions, celle par exemple de « rôle social », selon laquelle la situation des femmes est un fait social, non naturel ni inéluctable. Ce point est déterminant au regard du féminisme de George Sand.

\section{Le féminisme de George Sand et la formation de ses idées religieuses}

De son vivant, George Sand fut considérée comme trop féministe par les uns, pas assez selon d'autres. La question reste posée de nos jours, comme le démontrent les titres de quelques-uns des articles publiés dans "Le Magazine Littéraire » de mai 2004 déjà cité : "Femme ? La question inévitable» de Christine Planté, et de Michelle Perrot "George Sand n'a pas trahi le féminisme » (17). Pour ces deux auteurs, si la baronne Dudevant ne fut pas à la pointe des premiers combats féministes, ses romans ont à l'évidence posé clairement les problèmes, et à titre personnel, elle a su préserver ses droits en obtenant une séparation d'abord amiable, puis judiciaire d'avec son mari Casimir Dudevant. Grâce à ses talents d'écrivain, elle démontre qu'une femme peut obtenir son autonomie financière, chose rare à l'époque.

L'accueil réservé fait par George Sand aux mouvements féministes peut s'expliquer par des motifs conjoncturels, d'une part, et par les lacunes que comportait l'éducation des femmes à cette époque. Il n'est pas sans intérêt de noter que les relations de George Sand et de Flora Tristan, dont les idées étaient proches des siennes à bien des égards ne furent pas toujours cordiales. En 1837, Flora Tristan, dans les Pérégrinations d'une paria, reproche à George Sand la prudence qui l'amène à formuler ses critiques de la condition féminine sous le voile de la fiction romanesque, et à l'abri d'un pseudonyme masculin (18). Cette divergence de points de vue n'empêche pas les deux femmes d'entrer en relations. Mais George Sand aura une attitude de plus en plus réservée à l'égard de Flora, à qui elle reproche son exaltation de propagandiste, ainsi qu'un excès de confiance dans ses idées qu'elle qualifie d' «enfantillages » (19). La controverse de George Sand avec Eugénie Niboyet, en 1848, est mieux connue et amène George Sand à préciser ses positions. Le 16 avril 1848, Eugénie Niboyet proposait dans le journal La voix des femmes, la candidature de George Sand aux élections de l'Assemblée Constituante. Celle-ci répond par un désaveu le 8 avril suivant dans une lettre au rédacteur de La Réforme et à celui de La Vraie République. Elle regrette que son nom ait été avancé sans qu'elle ait été consultée : "Je ne puis permettre que, sans mon aveu, on me prenne

(16) Léon Abensour, op. cit., 1979 (1921), p. 168.

(17) Le Magazine littéraire, op. cit, pp. 50-57.

(18) Michaud, 1985, pp. 202-203.

(19) Idem, pp. 204-205. 
pour enseigne d'un cénacle féministe avec lequel je n'ai jamais eu la moindre relation, agréable ou fâcheuse » (20).

Pour George Sand, les femmes ne peuvent, en l'état, participer à la vie politique, en raison du type d'éducation qui leur est donnée, et de leur statut matrimonial. Cette situation n'est peut-être pas définitive, mais il faudra pour qu'elle évolue que les structures de la société se modifient en profondeur. Le héros du roman Isidora, Jacques Laurent se donne pour tâche de « régler les rapports de l'homme et de la femme dans la société, dans la famille, dans la politique » (21). L'éducation, les mœurs, les coutumes sont à l'origine du statut actuel des femmes. $\mathrm{Si}$ ces facteurs étaient modifiés, il se pourrait «que les aptitudes de l'un ou de l'autre sexe fussent complètement modifiées » (22). Au fil des années, les idées de George Sand en ce qui concerne les femmes se modifieront peu. Elle indique dans sa correspondance que l'amour maternel prévaudra toujours sur n'importe quelle considération, notamment dans une lettre à Édouard de Pompéry en date du 23 décembre 1864 : «Je vois la femme à jamais esclave de son cœur et de ses entrailles » (23). Le féminisme de George Sand doit être à présent confronté à la formation de ses idées religieuses, qui sont la résultante d'influences contrastées.

\section{Une première éducation soumise à des influences contrastées}

Aurore fut élevée principalement par sa grand-mère, Madame Dupin de Francueil, laquelle demeurait attachée aux idées du XVIII siècle. Elle mit en garde sa petitefille contre les dogmes et les superstitions, en dépit de quelques concessions à l'esprit du temps, lors des premières années de la Restauration. Aurore fit sa première communion après une rapide instruction religieuse. Toutefois, en dépit de cette atmosphère peu propice aux convictions religieuses, l'enfant s'était créé une divinité, Corambé, tantôt homme, tantôt femme, qui tenait le milieu entre le christianisme et les dieux de l'Iliade et de l'Odyssée (24). Martine Reid, dans un ouvrage récent, démontre l'importance de cet épisode, au regard de l'histoire familiale de la romancière (25). Lorsqu'elle était auprès de sa mère, née SophieVictoire Delaborde, d'origine beaucoup plus modeste, l'enfant avait l'exemple d'une piété non conformiste. Après un séjour de deux ans au couvent des Augustines anglaises à Paris, la future George Sand rentre à Nohant auprès de sa grand-mère, puis épouse Casimir Dudevant après la mort de celle-ci. Au contact des réalités de l'existence, les convictions religieuses acquises au couvent des Augustines anglaises à la suite d'une expérience mystique vont s'effriter progressivement. La future romancière se sépare de son mari et s'installe à Paris en compagnie de Jules Sandeau au début de l'année 1831. Les héroïnes de ses premiers romans, Indiana, Valentine, Lélia se réfèrent à un Dieu juste et bon, et non à celui des religions officielles, que les hommes ont créé à leur image (26). Après ce panorama de

(20) SAND, 1997, pp. 529-542.

(21) SAND, 1998, (1846), p. 9.

(22) Idem, p. 26.

(23) SAND, Correspondance, 1984, p. 629.

(24) SAND, 1970, $3^{\mathrm{e}}$ partie, chapitre VIII, pp. 812-813.

(25) REID, 2003.

(26) SAND, « Romans $1830 »$, in Indiana, 1991, p. 132. 
l'ambiance intellectuelle qui a présidé à la formation de George Sand, il convient de décrire la synthèse faite par la romancière de ces différents courants de pensée.

\section{La synthèse entre féminisme et religion dans la pensée de George Sand}

\section{Vers le socialisme chrétien}

Bien introduite dans les milieux littéraires de son temps, George Sand, après une période de doute et de scepticisme va rencontrer des hommes dont les idées lui rendront confiance et espoir : Félicité de Lamennais (1788-1854) et le philosophe Pierre Leroux (1797-1871). George Sand s'intéresse assez tôt aux idées de Félicité de Lamennais, un prêtre qui, après avoir soutenu des thèses ultra-conservatrices dans un ouvrage en plusieurs volumes intitulé Essai sur l'indifférence en matière de religion s'orientera vers un christianisme social qui s'exprime dans Paroles d'un croyant, ouvrage publié au début de l'année 1834, condamné par l'encyclique Singulari Nos le 7 juillet 1834, ce qui radicalisera la rupture de Lamennais avec l'Église. En avril 1835, à l'occasion du «procès monstre » George Sand aura l'opportunité de rencontrer les deux penseurs, qui se proposaient d'assurer la défense des accusés (27) De Pierre Leroux, ancien ouvrier imprimeur, la romancière retient les thèses du progrès continu et de l'égalité, qui selon lui constituent le fond de toutes les croyances religieuses (28) (29).

Spiridion, publié en 1839, retrace la crise spirituelle de Lamennais, ainsi que ses difficultés avec l'Église. L'ouvrage a pour cadre un monastère italien à la veille de la Révolution. Un moine, Alexis, et un novice, Angel, s'intéressent aux idées du fondateur du couvent, Spiridion, qui, après avoir adhéré successivement aux religions institutionnelles de son temps, a découvert l'Évangile Éternel de Joachim de Flore (1132-1202). Ce dernier a eu une vision mystique lors d'un voyage en Terre Sainte, et à partir de différents textes bibliques dont l'Apocalypse, annonce pour l'année 1260 l'Ère du Saint-Esprit, qui succède à celle du Père, correspondant à l'Ancien Testament, et à celle du Fils, qui recouvre la révélation chrétienne. Cette ère nouvelle verra naître L'Église de saint Jean, laquelle n'aura plus besoin de rites, ni de dogmes car la vérité apparaîtra clairement à chacun (30). De toute évidence, ces idées corroborent les thèses de Pierre Leroux sur le progrès continu de l'humanité.

Le joachimisme sera également la toile de fond d'un dialogue philosophique, Les Sept Cordes de la Lyre, composé par George Sand pendant une interruption de la rédaction de Spiridion, ce qui explique la parenté d'idées véhiculées par les deux textes. Les Sept Cordes de la Lyre s'inspire d'un texte de Pierre-Simon Ballanche (1776-1847), intitulé Orphée (31). Mais le titre évoque Joachim de Flore qui avait

(27) HAMON, 2001.

(28) Leroux, 1978, p. 25.

(29) Leroux, 1985 (1840), p. 203.

(30) SAND, 2000 (1842), p. 290.

(31) Ballanche, 1967, pp. 419-550. 
composé un ouvrage intitulé Le Psaltérion à dix cordes et où se retrouve également une atmosphère d'Apocalypse, puisque l'héroïne, Hélène, monte sur la tour de la cathédrale et tire des sons de la lyre : les passants y voient un fait miraculeux. Une vieille dame affirme «l'archange de la cathédrale a joué de la trompette » (32), ce qui renvoie aux anges à la trompette de l'Apocalypse, dont les interventions ponctuent la venue de la fin des temps.

Le long roman intitulé Consuelo, suivi de La comtesse de Rudolstadt permettra à l'auteur de préciser ce qu'elle attend des idées véhiculées par certains mouvements hétérodoxes de la fin du Moyen Âge : Hussites, Vaudois, Lollards, etc... La cantatrice Consuelo séjourne en Bohême, au château des Géants, propriété de la famille de Rudolstadt, dont le dernier représentant, Albert, se dit le descendant par sa mère et se croit la réincarnation du chef hussite Jean Ziska (33). Consuelo se lie d'amitié avec Albert, lequel lui expose ses convictions religieuses : la négation de l'enfer, la réhabilitation de Satan et de la chair, la communion, gage et symbole de l'égalité entre les hommes (34).

\section{Les thèmes religieux émancipateurs}

Entre la rédaction des deux parties du roman, George Sand rédige une biographie du chef hussite Jean Ziska, où elle précise les causes de son intérêt pour les mouvements religieux hétérodoxes : «voilà ce que c'est que l'hérésie (...) : une idée essentiellement chrétienne dans son principe évangélique, révolutionnaire dans ses tentatives et ses réclamations ». La romancière ajoute que toutes ces sectes ne constituent qu'une seule et même histoire, celle du joannisme, c'est-à-dire l'interprétation et l'application de l'Évangile fraternel et égalitaire de saint Jean. C'est la «doctrine de l’Évangile Éternel, ou de la religion du Saint-Esprit, qui remplit tout le Moyen Âge et qui est la clé de toutes ses convulsions, de tous ses mystères » (35). Les femmes sont particulièrement intéressées à cette histoire, car elles sont toutes «des hérétiques, toutes vous protestez dans votre cœur (...). Vous êtes toutes par nature et par nécessité les disciples de saint Jean et de saint François, et des autres grands apôtres de l'idéal » (36). Les femmes, en effet, de par leur statut matrimonial, peuvent être assimilées à des pauvres puisque privées de la disposition de leurs biens.

La négation de l'enfer est présente dans toute l'œuvre de George Sand. Sa première éducation l'y avait préparée, comme elle l'indique dans son autobiographie (37). La croyance aux peines éternelles de l'enfer semble à George Sand incompatible avec la bonté divine. Elle exprime cette idée dans sa correspondance également. Elle écrit le 21 août 1863 à un pasteur, Adolphe Schaeffer, qui lui avait transmis son Essai sur l'avenir de la tolérance: "Détruisons ce faux Dieu (...) pour que les esprits éclairés et les âmes aimantes se rallient à votre Église, il faudra bien l'ouvrir toute grande cette porte au-delà de laquelle on peut voir le vrai Dieu (...). Prenez-y garde, les nouvelles générations n'y passeront pas, si l'enfer est

(32) SAnd, Les Sept Cordes de la Lyre, in Euvres complètes, 1980, p. 134.

(33) SAND, tome 1, 1959, pp. 319-320.

(34) Idem, tome 2, 1959, pp. 14-22.

(35) SAnd, Jean Ziska, in Euvres complètes, Tome XIX, 1980, pp. 25-30.

(36) Idem, p. 19

(37) SAnd, Histoire de ma vie, op. cit., 1970, p. 24 
au seuil » (38). Ce refus de la croyance à l'enfer devient un instrument d'émancipation en général et des femmes en particulier. L'auteur, dans la préface de l'édition de 1871 de son roman Mademoiselle La Quintinie indique clairement que la crainte de l'enfer est un moyen d'oppression: «N'est-il pas bon que les paysans, les enfants et les femmes soient menés par la peur ?» (39).

Le caractère divin de l'amour, thème qui apparaît dès les premiers romans de George Sand est un autre moyen d'émancipation. L'amour vrai ne peut ni se commander, ni être extirpé par la volonté de l'homme, comme indiqué dans le roman de Valentine: " $\mathrm{Si}$ l'amour était un sentiment qui se calcule et se raisonne (...). Mais ce qui fait l'immense supériorité de celui-là sur les autres, ce qui prouve son essence divine, c'est que l'homme n'en peut disposer, c'est qu'il ne l'accorde pas plus qu'il ne l'ôte par un acte de volonté »(40). Tout au long de ce même roman, les barrières sociales qui empêchent le mariage de Bénédict et de Valentine vont être à l'origine de catastrophes, suicides, morts violentes, qui forment la trame du récit. Dans la Comtesse de Rudolstadt, Wanda, la mère d'Albert, chargée d'initier Consuelo aux idées de la société des Invisibles, se fera la théoricienne du caractère divin de l'amour. Résister à ce sentiment revient donc à désobéir à Dieu. Les enfants qui naissent de telles unions, marqués « du sceau de la désobéissance », sont porteurs d'une tare quelconque (41). Après avoir retracé le parcours intellectuel de George Sand jusqu'au milieu du siècle, il convient de rechercher ce que devinrent ses convictions au fil du temps et suite à leur confrontation aux réalités de l'existence.

\section{La sagesse de l’âge mûr}

Le socialisme chrétien de George Sand ne semble pas avoir survécu bien longtemps aux désillusions consécutives à la révolution de 1848. Désormais, la romancière mettra l'accent sur les efforts individuels, le travail, le rapprochement entre les catégories sociales par l'abandon de leurs préjugés, ce qu'elle exprime par exemple dans La Ville Noire (42). Dans un conte écrit en 1873, Le géant Yéous, le travail individuel, la patience, permettent de lutter contre de prétendues fatalités (43). Néanmoins, le fond d'idées religieuses de George Sand, se ramène essentiellement à la croyance en la bonté de Dieu et à une vie future, comme la romancière l'explique à différentes reprises dans sa correspondance, notamment dans une lettre à Victor Hugo, en date du 17 avril 1862 : «Je plaide la cause de mon utopie : le bien possible dès cette vie sans souffrances insupportables, le beau certain dans l'autre vie pour qui a su se l'assurer » (44). Ses idées s'enrichissent et se diversifient au fil des années. Elle découvrira en 1854 un livre de l'ex-saintsimonien Jean Reynaud, qui collaborait avec Pierre Leroux à L'Encyclopédie nouvelle, l'essai intitulé Terre et Ciel, qui évoque la transmigration des âmes ; l'orphisme, le celtisme (45). La métempsycose sera le thème d'un Conte d'une

(38) SAnd, Correspondance, op. cit., tome XVII, 1984, p. 39.

(39) SAND, «Préface» in Mademoiselle..., 1863, édition de 1971, p. VII.

(40) SAND, Valentine in George SAND, Romans 1830, 1991, p. 269.

(41) SAND, La comtesse de Rudolstadt, Tome 3, 1959c, p. 383.

(42) SAND, La Ville Noire, 2000, (1861)

(43) SAnd, Le Géant Yéous, in George SAnd, Contes d'une grand-mère, 1975, Tome 1, p. 315.

(44) Sand, Correspondance, op. cit., Tome XVII, 1984, p. 33.

(45) Chalaye, 2000, pp. 16-29. 
grand-mère, intitulé Le chien et la fleur sacrée (46), qui comporte des considérations sur l'évolution cosmique, caractérisée par la spiritualisation de la matière et le progrès continu de l'univers.

À la fin de sa vie, George Sand n'a plus de liens avec l'Église catholique. Son roman Mademoiselle La Quintinie comporte des pointes anticléricales, qui ne sont pas sans évoquer le programme politique ultérieur de la Troisième République, visant à réduire les ingérences de clercs, de religieux tant dans la vie privée que dans la politique. Néanmoins, apparaissent de manière récurrente dans ses romans, des prêtres, des religieux qui, tels le frère Nicolas des Maîtres Sonneurs (47), le chanoine de Saint-Étienne dans Consuelo, l'abbé Féline dans Simon (48) exercent une action utile et bienfaisante. À l'occasion du mariage de son fils Maurice en 1862, et lors de la naissance du premier enfant de celui-ci, George Sand aura l'occasion d'entrer en correspondance avec des ministres du culte protestant. Elle se sent plus proche de leurs croyances que de celles de l'Église catholique, mais ne peut y adhérer totalement. Lorsqu'elle meurt en 1876, elle n'a donné aucune instruction pour ses obsèques. À l'instigation de sa fille Solange, George Sand sera enterrée selon les rites de l'Église catholique, ce qui donnera lieu à d'innombrables commentaires (49).

\section{George Sand et les religions « amies du droit de la mère »}

L'attitude de George Sand face aux Églises chrétiennes et à leurs ministres ne sont pas les seuls éléments à prendre en compte pour définir ses idées religieuses. Comme tend à le démontrer son intérêt pour l'ouvrage précité de Jean Reynaud, l'enquête doit s'étendre aux religions orientales, au celtisme, aux mythologies païennes de l'Antiquité. C'est dans ces courants que la romancière puisera l'idée de la femme guide, inspiratrice, et de ses fonctions d'initiatrice. Un de ses contes, Le Château de Pictordu, véhicule une image de la Grande Déesse proche des analyses de Robert Graves précitées. Monsieur Flochardet et sa fille Diane sont contraints de passer une nuit au château de Pictordu, situé dans un « désert de forêts et de montagnes » du Gévaudan (50). Ce château abandonné est réputé hanté. La fillette se réveille et voit « une grande belle clarté bleue, comme celle de la lune, traverser la salle, et dans ce rayon de lumière douce, elle vit que la danseuse antique avait quitté la muraille et venait à elle ». «Ne croyez point qu'elle eut peur, c'était une forme exquise. Sa robe faisait mille plis gracieux sur son beau corps et semblait semée de paillettes d'argent: une ceinture de pierreries retenait les pans de sa tunique légère ; un voile de gaze brillante était roulé sur sa chevelure qui s'échappait en tresses blondes sur ses épaules blanches comme neige. On ne pouvait distinguer son visage à travers cette gaze, mais il en sortait comme deux pâles rayons à la place des yeux » (51).

(46) SAND, Le chien et la fleur sacrée, in Contes d'une grand-mère, tome II, 1975, pp. 63-142.

(47) SAND, 1979, pp. 240-243

(48) SAnd, Simon, Tome XXXI des Euvres Complètes, (reprint de l'édition Lévy de 1863-1926), Genève, Slatkine, 1980, p. 9.

(49) KarÉnine, Tome IV, (1848-1876), (1926), 2000, pp. 599-631.

(50) SAND, Contes d'une grand-mère, 1976, pp. 4-5.

(51) Idem, pp. 21-22 
Cette représentation est fort proche du texte d'Apulée, cité par Robert Graves dans La Déesse Blanche, comme le démontre cet extrait : "Quelques instants plus tard, alors que mes yeux venaient de se fermer, m'apparut, s'élevant de la mer, une divine et vénérable face que les dieux eux-mêmes eussent adorée (...). Au milieu de son front était un petit cercle plat, une sorte de miroir ou plutôt de lune à cause d'une lumière qu'il projetait » (52). Bien des personnages féminins jouent un rôle de guides ou d'initiatrices dans les écrits de George Sand. À cet égard, le conte intitulé Laura. Voyage dans le cristal est particulièrement significatif. C'est Laura qui initie Alexis et qui tout au long du voyage va stimuler sa progression. Sa présence, à la fin du récit allégorique dissipe les rêves de richesse et de puissance suscités par Nasias. Alexis reprend contact avec la réalité et demande Laura en mariage (53).

Dans «Le Nuage rose», tante Colette démontre à Catherine qu'il ne faut compter ni sur les miracles, ni sur le mystère, et que seuls le travail, le courage, l'économie peuvent rendre la vie plus agréable et plus facile. Madame Colette explique : "Le nuage rose, c'était mon caprice, ma fantaisie, mon mauvais destin. Je l'ai mis sur ma quenouille, et le travail, le beau et bon travail a fait de l'ennui un fil si léger que je ne l'ai plus senti » (54). Dans le roman Jeanne, les emprunts au paganisme et au celtisme sont patents, aussi bien dans le décor, où des mégalithes sont longuement décrits (55), que dans la psychologie des personnages, puisque l'héroïne, Jeanne, est présentée comme une «Isis gauloise » (56). George Sand a donc su tirer parti dans son œuvre de la redécouverte par les romantiques des cultes féminins, ainsi que de leur caractère émancipateur.

\section{Conclusion}

Il est donc permis de conclure que George Sand, qui n'a pu connaître de son vivant les thèses de Bachofen, a eu l'intuition des « religions amies du droit de la mère » sous l'impulsion des religiosités romantiques, lesquelles redécouvraient sous de multiples formes les cultes féminins. Mais George Sand ne pouvait totalement tirer parti de ces découvertes. Ce n'était pas sans motifs qu'elle affirmait, notamment à l'occasion des polémiques de 1848, que la modification du statut des femmes, leur participation à la vie politique étaient subordonnées à de profondes transformations de la société. En effet, de par leur statut civil et leur éducation, ce dont elles n'étaient pas responsables, elles étaient mal préparées à une intervention dans la politique active. De profonds changements s'avéraient donc préalablement nécessaires. Mais George Sand considérait en outre que la place des femmes dans la société était conditionnée dans une certaine mesure par des facteurs naturels. Ce ne fut que grâce aux progrès de certaines sciences humaines et sociales, telles que l'ethnologie, la sociologie, l'étude comparée des religions qu'il apparut que le rôle

(52) GRAVES, 1979, p. 79.

(53) SAND, 1977 (1865).

(54) SAND, «Le nuage rose », in Contes d'une grand-mère, Tome I, 1975, p. 199.

(55) SAND, 1986, pp. 87-88.

(56) Idem, p. 169. 
des femmes dans la société n'était pas exclusivement un fait de nature, puisqu'il varie en fonction des temps et des lieux. George Sand eut le mérite de mesurer le problème, puis d'avoir l'intuition que dans l'avenir, les données pourraient en être substantiellement modifiées.

Marie-Reine RENARD

Chercheur indépendant

\section{BIBLIOGRAPHIE}

ABENSOUR Léon, Histoire générale du féminisme des origines à nos jours, Genève, Slatkine, 1979 (1921).

BACHOFEN Joahann-Jakob, Das Mutterrecht, (traduit et publié par le Groupe français d'études féministes), Paris, Groupe français d'études féministes, 1903.

BACHOFEN Joahann-Jakob, Du droit de la mère au patriarcat, pages choisies par Adrien Turel, Agora, éditions de l'Aire, 1980 (1938).

BACHOFEN Joahann-Jakob, Le droit maternel. Recherches sur la gynécocratie de l'Antiquité dans sa nature religieuse et juridique. (traduit de l'allemand et préfacé par Étienne Barilier) Lausanne, l’Âge d'Homme, 1993 (1861).

BALLANCHE Pierre-Simon, Orphée, in le reprint des Euvres complètes de 1833, Genève, Slatkine, 1967.

BÉNICHOU Paul, Le Temps des prophètes. Doctrines de l'âge romantique, Paris, Gallimard, 1977.

BERNARD-GRIFFITHS Simone, Ville, campagne, nature dans l'œuvre de George Sand, (textes réunis et présentés par Simone Bernard-Griffiths), Clermont-Ferrand, Presses Universitaires Blaise Pascal, 2002.

BOISDEFFRE Pierre de, George Sand à Nohant, sa vie, sa maison, ses voyages, ses demeures. (préface de Françoise Chandernagor), Paris, Pirot, 2000.

BOYER Philippe, Le romantisme allemand, Paris, MA Éditions, 1985.

CHALAYE Gérard, "La mémoire des étoiles : George Sand-Jean Raynaud. 1854-1855 », in Les Amis de George Sand, no 22, 2000, pp. 16-29.

CHARLETY Sébastien, Histoire du saint-simonisme, (1825-1864), Paris, Gonthier, 1965 (1931),

CHEVEREAU Anne, George Sand, du catholicisme au paraprotestantisme?, (préface de Jean Baubérot), Antony, 1988.

CELLIER Léon, GUICHARD Léon, «L'occultisme dans Consuelo et la comtesse de Rudolstad » in George SAND, Consuelo, Tome I, Paris, Garnier, 1959a (1842-1844) pp. XLVII-LXXVIII.

CHRISTOPHE Paul, George Sand et Jésus, Paris, Cerf, 2003.

DUFOUR Hortense, George Sand la somnambule, Monaco, Éditions du Rocher, 2002.

EVANS David Owen, Le socialisme romantique. Pierre Leroux et ses contemporains, (préface d'Édouard Dolléans), Paris, Rivière, 1948.

GEBHART Émile, L'Italie mystique. Histoire de la renaissance religieuse au Moyen Âge, Paris, Hachette, 1908.

GEORGOUDI Stella, «Étude sur Bachofen » in Georges DUBY, Michelle PERROT, éds., Histoire des femmes en Occident, I. L'Antiquité, Paris, Éditions Perrin, 2002, pp. 585 à 602 (coll. « Tempus »).

GRAVES Robert, La déesse blanche. Un mythe poétique expliqué par l'histoire. (Traduit de l'anglais par Guy Trévoux), (première édition: The White Goddess, a Historical Grammar of Poetic Myth, Faber and Faber, 1948), Monaco, Éditions du Rocher, 1979. 
HAMON Bernard, George Sand et la politique, (préface de Michelle Perrot), Paris, L'Harmattan, 2001.

HOFFMANN E.T.A., « Le Vase d'or », in Contes à la manière de Callot tirées du journal d'un voyageur enthousiaste, (traduction André Espiau de la Maëstre), Paris, Gallimard, 1964, pp. 241-365.

HOOG-NAGINSKI Isabelle, George Sand. L'écriture ou la vie, (traduit par Nadine Dormoy), Paris, Champion, 1999.

KARÉNINE Wladimir, George Sand, sa vie et ses æeuvres, 4 volumes, Genève, Slatkine, 2000 (1899 à 1926).

LACASSAGNE Jean-Pierre, Histoire d'une amitié. Pierre Leroux et George Sand, d'après une correspondance inédite, 1835/1866, Paris, Klincksieck, 1973.

LECLERCQ Pierre-Robert, George Sand, les années Aurore, Paris, Anne-Carrière, 2004.

Le Magazine Littéraire, $\mathrm{n}^{\mathrm{O}}$ 431, mai 2004.

LEO Gerhard, Flora Tristan, La révolte d'une paria, Paris, Éditions de l'Atelier, 1994.

LEROUX Pierre, Trois discours philosophiques, dans CEuvres (1825-1850), (Reprint de l'édition de Paris, 1850-1851), Genève, Slatkine, 1978.

LEROUX Pierre, La grève de Samarez. Poème philosophique, 2 volumes, (introduction et notes de JeanPierre Lacassagne), Paris, Klincksieck, 1979.

LEROUX Pierre, De l'humanité, (texte revu par Miguel Abensour et Patrice Vermeren), Paris, Fayard, 1985 ((1840).

LUBAC Henri de, La postérité spirituelle de Joachim de Flore, 2 volumes, Paris, Lethielleux, 1979 et 1981 (sur George Sand et Pierre Leroux, Tome II, pp. 135-188).

MAUROIS André, Lélia ou la vie de George Sand, Paris, Hachette, 1952.

MACHEREY Pierre, «Un roman panthéiste : Spiridion de George Sand », in Que pense la littérature, Paris, PUF, 1990, pp. 37-51.

MICHAUD Stéphane, «En miroir: Flora Tristan et George Sand », in Actes du premier colloque international Flora Tristan, Un fabuleux destin: Flora Tristan présenté par Stéphane Michaud, Éditions Universitaires de Dijon, 1985, pp. 202-203.

MORET Pierre, Le sentiment religieux chez George Sand, (Thèse présentée à la Faculté des Lettres de l'Université de Paris), Vigne, 1936.

PLATON, 1965 (-416), Le Banquet, (traduction et notes par E. Chambry), Paris, Garnier-Flammarion, 1964.

POMMIER Jean, George Sand et la vie monastique. Spiridion, Paris, Nizet, 1966.

POWELL, David A., Le siècle de George Sand, (textes réunis et présentés par Powell, avec l'assistance de Shira Malkin), Amsterdam-Atlanta, Rodopi, 1998.

PUECH Jules L., La vie et l'œuvre de Flora Tristan, (1803-1844), Paris, Rivière, 1925.

REID Martine, Signer Sand: L'œuvre et le nom, Paris, Belin, 2003.

RENAN Ernest, «Joachim de Flore et l'Évangile Éternel », in Nouvelles Études d'Histoire Religieuse, Tome VII des Euvres complètes, Paris, Calmann Lévy, 1955, pp. 853-918.

SAND George, Mademoiselle La Quintinie, Paris, Librairie Nouvelle, 1871 (1863).

SAND George, Consuelo, Tome I, (texte établi, annoté et présenté par Léon Cellier et Léon Guichard), Paris, Garnier, 1959a (1842-1844).

SAND George, Consuelo, Tome II, (texte établi, présenté et annoté par Léon Cellier et Léon Guichard), Paris, Garnier, 1959b (1842-1844).

SAND George, La comtesse de Rudolstadt, Tome III, Paris, Garnier, 1959c (1842-1844).

SAND George, Histoire de ma vie, (texte établi, présenté et annoté par Georges LUBIN), Paris, Gallimard, 1970.

SAND George, Contes d'une grand-mère, (texte établi par Georges Lubin), Paris, Éditions d'Aujourd'hui, 2 volumes, 1975 (1873).

SAND George, Légendes rustiques : les esprits et les visions de la nuit dans les campagnes, Paris, Marabout, 1975 (1858).

SAND George, Laura. Voyage dans le cristal, (introduction par Gérald Schaeffer), Paris, Nizet, 1977 (1865).

SAND George, Les Maîtres sonneurs, (édition présentée, établie et annotée par Marie-Claire Bancquart), Paris, Gallimard, 1979. 
SAND George, Correspondance, Tome XVII, (édition Georges Lubin), Paris, Garnier, 1984.

SAND George, Les Sept Cordes de la Lyre, in Euvres complètes, Tome XXXI du reprint de l'édition Lévy de 1863 à 1926, Genève, Slatkine, 1980.

SAND George, Jean Ziska in Euvres complètes, Tome XIX du reprint de l'édition Lévy de 1863 à 1926 , Genève, Slatkine, 1980.

SAND George, Simon, in Euvres Complètes, Tome XXXI, (reprint de l'édition Lévy de 1863-1926), Genève, Slatkine, 1980, p. 9

SAND George, La Petite Fadette, (texte établi, présenté et annoté par Pierre Salomon et Jean Mallion), Paris, Garnier, 1981 (1849).

SAND George, La Mare au Diable. (préface, commentaires et note de Pierre de Boisdeffre), Paris, Le Livre de Poche, 1984 (1845).

SAND George, Jeanne, (préface de Simone Vierne), Meylan, Éditions de l'Aurore, 1986.

SAND George, Romans 1830, in Indiana, (préface de Marie-Madeleine Fragonard), Paris, Omnibus, 1991.

SAND George, Valentine, in Romans 1830, (préface de Marie-Madeleine Fragonnard), Paris, 1991.

SAND George, Politique et Polémiques (1843-1850), (présentation Michelle Perrot), Paris, Imprimerie des Éditions Nationales, 1997.

SAND George, Isidora, Paris, Findakly, 1998 (1846).

SAND George, La ville Noire, (texte établi, présenté et annoté par Jean Courrier), Clermont-Ferrand, Borée, 2000 (1861).

SAND George, Spiridion, (avant-propos d'Oscar A. Haac, introduction de Michèle Hecquet), Genève Slatkine, 2000 (1842).

SAND George, Les Femmes, (textes choisis et présentés par Huguette Bouchardeau), Nîmes, HB, 2003 (1848).

SAND George, Journal d'un voyageur pendant la guerre, (édition présentée par Michelle Perrot), Paris, La Castor Astral, 2004 (1871).

SAND George, Consuelo / La comtesse de Rudolstadt, (présentation par Nicole Savy ; texte établi et annoté par Damien Zanone), Paris, Laffont, 2004.

SAND George, Histoire de ma vie, (édition établie, présentée et annotée par Martine Reid), Paris, Gallimard, 2004 (1854-1855).

SHELLEY Percy B., La Reine Mab, dans Euvres poétiques complètes, Tome 1, (traduit par F. Rabbe), Paris, Stock, 1907.

THIBERT Marguerite, Le féminisme dans le socialisme français de 1830 à 1850, Paris, Giard, 1926.

VAN DIJK Suzan, « George Sand et les mouvements d'émancipation féminine : lectures étrangères » in Le siècle de George Sand, (textes réunis par David A. Powell, avec l'assistance de Shira Malkin), Amsterdam-Atlanta, Rodopi, 1998.

VERMEYLEN Pierre, Les idées politiques et sociales de George Sand, Bruxelles, Éditions de l'Université de Bruxelles, 1984.

VIATTE Auguste, Les sources occultes du romantisme, 2 volumes, Paris, Champion, 1979 (1927). 
Résumé

À l'occasion de la commémoration du bicentenaire de la naissance de George Sand, et dans la perspective de l'année de la laïcité, il est intéressant d'analyser les rapports qui existent entre sentiment religieux et émancipation féminine dans l'œuvre de George Sand, laquelle constitue par ailleurs un bon reflet des idées de son temps.

Le féminisme de George Sand, toujours sujet à controverses, en marque les limites intellectuelles au XIX ${ }^{e}$ siècle. L'émancipation féminine, en effet, ne sera effective que grâce à l'avancée des sciences humaines, ce dont la romancière aura l'intuition, lorsqu'elle affirmait que la participation des femmes à la vie politique était subordonnée à de profonds changements des structures de la société, et en se référant aux religions "amies du droit de la mère».

\section{Abstract}

On the occasion of the commemoration of the bicentennial of George Sand's birth an the context or the year of "laïcité, an analysis of the relationships between religious feeling and emancipation of women in the works of George Sand is of the interest, her works being also a good indication of the prevailing ideas of her time.

George Sand's feminism, always the subject of controversy, marks the intellectual limits of the nineteenth century. Emancipation of women will in fact only become a reality through progress in human science. The novelist had perceived this when she stated that women's participation in political life was linked to profound changes in society's structures, and when she referred to religions "favouring mothers' rights".

Resumen

Con motivo de la commemoraciòn del bicentenario del nacimiento de George Sand, y teniendo en perspectiva el año del laicismo, es interesante analizar las relaciones que existen entre el sentimiento religioso y la emancipaciòn femenina en la obra de George Sand que, ademàs, constituye un espejo muy adecuado de la ideas de su época.

El feminismo de George Sand, sujeto a frecuentes controversias, le impone limites sus intelectuales durante el siglo XIX, ya que la emancipaciòn feminina sòlo serà efectiva gracias al progreso de las ciencias humanas, tal como la novelista lo habia intuido cuando afirmaba que la participatiòn de las mujeres en la vida politica estaba subordinada a profundos cambios de las estructuras e la sociedad, refirièndose a las religiones "amigas del derecho de la madre». 